\title{
Modulated two-level system: Exact work statistics
}

\author{
Gatien Verley, ${ }^{1}$ Christian Van den Broeck, ${ }^{2}$ and Massimiliano Esposito ${ }^{1}$ \\ ${ }^{1}$ Complex Systems and Statistical Mechanics, University of Luxembourg, L-1511 Luxembourg, G.D. Luxembourg \\ ${ }^{2}$ Hasselt University, B-3590 Diepenbeek, Belgium
}

(Received 1 August 2013; published 27 September 2013)

\begin{abstract}
We consider an open two-level system driven by a piecewise constant periodic field and described by a rate equation with Fermi, Bose, and Arrhenius rates, respectively. We derive an analytical expression for the generating function and large deviation function of the work performed by the field and show that a work fluctuation theorem holds.
\end{abstract}

DOI: 10.1103/PhysRevE.88.032137

PACS number(s): 05.70.Ln, 05.40.-a, 05.20.-y

\section{INTRODUCTION}

According to standard thermodynamics, the amount of work needed to bring a system in contact with an heat bath at temperature $T$ from one equilibrium state to another one is at least the corresponding difference in equilibrium free energy $\Delta F^{\mathrm{eq}}$. This result is a direct consequence of the second law. This traditional formulation has been developed for macroscopic systems where the measured work $W$ essentially coincides with its expectation value $\langle W\rangle$. Over the past two decades, this issue has been revisited for the case of driven nonmacroscopic systems, with surprising theoretical consequences. For a small system, the work $W$ will fluctuate from one experiment to the other and the full distribution of work $P_{W}$, rather than solely the average, becomes the experimentally accessible quantity of interest. Starting from basic physical principles and considering a system initially at equilibrium with a single heat bath at inverse temperature $\beta=1 /\left(k_{B} T\right)$, set to unity throughout the paper, one can derive the Jarzynski equality $[1,2]\langle\exp (-\beta W)\rangle=\exp \left(-\beta \Delta F^{\mathrm{eq}}\right)$ and the Crooks work theorem [3-8] $P_{W} / \bar{P}_{-W}=\exp \left\{\beta W-\beta \Delta F^{\mathrm{eq}}\right\}$. In this last fluctuation theorem, the bar refers to the experiment with time-reversed driving. These results imply the standard thermodynamic inequality $\langle W\rangle-\Delta F^{\mathrm{eq}} \geqslant 0$. In light of these tantalizing developments, there has been an obvious interest in verifying that distributions of work indeed satisfy these equalities, and if possible, to calculate their explicit form. This has been achieved in a number of scenarios including models with Langevin dynamics [9-17], (granular) gas models [18-20], mean-field models [21,22], and a discrete (toy) model [23]. $P_{W}$ has also been measured experimentally [24-30] and numerically [31-34].

Somewhat surprisingly, the calculation of the work distribution is notoriously difficult for one of the prototype models of statistical mechanics, namely a two-state system driven by a modulated field [35-37]. Even the case of periodic modulation is challenging as it turns out to be, mathematically speaking, closely related to the parametric oscillator. The main purpose of this paper is to provide an exact analytical solution for the work distribution of a two-level system subjected to periodic piecewise constant modulation. Our method to obtain the work fluctuations is general and can be extended to other problems involving discontinuities in the driving. In the case of the two-state model, we show that a work fluctuation theorem (FT) holds, we discuss the similarities and differences between
Fermi, Bose, and Arrhenius rates, and explore various limiting regimes.

\section{PERIODICALLY MODULATED SYSTEM}

We consider a two-level system $\sigma= \pm 1$ subjected to an external field $h$ and coupled to a heat bath at temperature $T$. This model could describe, for example, a Brownian particle in a periodically modulated double-well potential [38-40], a single-level quantum dot subjected to a modulated electric field, or a photochromic defect in diamond similar to that of Ref. [41]. The energy change in the system obeys the first law of thermodynamics: the rate of change of the system energy $E=-h \sigma$ is the sum of a work flow $\dot{W}=-\dot{h} \sigma$ and a heat flow $\dot{Q}=-h \dot{\sigma}$; i.e., $\dot{E}=\dot{W}+\dot{Q}$. We will focus on the evaluation of the cumulated work

$$
W=-\int_{0}^{t} d t^{\prime} h\left(t^{\prime}\right) \sigma\left(t^{\prime}\right) .
$$

Due to its interaction with the heat bath, the system undergoes thermal transitions between its two states. Let $\omega_{-\sigma, \sigma}$ denote the probability per unit time for the system to flip from state $\sigma$ to state $-\sigma$. The resulting Markovian stochastic dynamics is characterized by a 2 by 2 transition rate matrix $\boldsymbol{L}$ with elements $L_{\sigma, \sigma^{\prime}}=-\sigma \sigma^{\prime} \omega_{-\sigma^{\prime}, \sigma^{\prime}}$, where

$$
\omega_{-\sigma, \sigma}=\omega(h) e^{-\sigma h} .
$$

These rates satisfy local detailed balance $[42,43]$

$$
\frac{\omega_{-\sigma, \sigma}}{\omega_{\sigma,-\sigma}}=\frac{p_{-\sigma}^{\mathrm{eq}}}{p_{\sigma}^{\mathrm{eq}}} \quad \text { where } \quad p_{\sigma}^{\mathrm{eq}}=e^{\beta \sigma h+\beta F^{\mathrm{eq}}} .
$$

Equation (2) includes as special cases, Arrhenius rates $\omega(h)=$ $\Gamma$, Fermi rates $\omega(h)=\Gamma /(2 \cosh (h))$, and Bose rates $\omega(h)=$ $\Gamma /(2|\sinh (h)|)$, where $\Gamma$ is a positive constant setting the time scale (and set to unity in our plots). The probability $p_{\sigma}$ to find the system in state $\sigma$ evolves according to the master equation

$$
\partial_{t} p_{\sigma}=\sum_{\sigma^{\prime}= \pm 1} L_{\sigma, \sigma^{\prime}} p_{\sigma^{\prime}} .
$$

For a constant value of the field $h$, it will eventually reach equilibrium $p_{\sigma}^{\mathrm{eq}}$, while for a time-periodic field driving it will eventually reach a periodic steady state [44].

The rate of change of the work, $\dot{W}=-\dot{h} \sigma$ in unit of $k_{B} T(\beta=1)$, is a deterministic function of the process $\sigma$. Hence, the joint set of variables $(\sigma, W)$ again defines a Markov 
process, and the corresponding joint probability $P_{\sigma, W}$ obeys the following evolution equation

$$
\partial_{t} P_{\sigma, W}=\sum_{\sigma^{\prime}= \pm 1} L_{\sigma, \sigma^{\prime}} P_{\sigma^{\prime}, W}-\partial_{W} \dot{W} P_{\sigma, W},
$$

with the initial condition, $P_{\sigma, W}(0)=\delta(W) p_{\sigma}(0)$, being the probability to be on state $\sigma$ with $W=0$ at time $t=0$. The probability distribution for the cumulated work follows by summation over the system states $P_{W}=\sum_{\sigma= \pm 1} P_{\sigma, W}$. By introducing the generating function

$$
G_{\mu}=\sum_{\sigma} G_{\sigma, \mu}, \text { where } G_{\sigma, \mu}=\int_{-\infty}^{\infty} d W e^{\mu W} P_{\sigma, W},
$$

one obtains from Eq. (5)

$$
\partial_{t} G_{\sigma, \mu}=\sum_{\sigma^{\prime}= \pm 1} L_{\sigma, \sigma^{\prime}}^{(\mu)} G_{\sigma^{\prime}, \mu},
$$

with $\boldsymbol{L}^{(\mu)}$ a matrix with elements $L_{\sigma, \sigma^{\prime}}^{(\mu)}=L_{\sigma, \sigma^{\prime}}-\dot{h} \mu \sigma \delta_{\sigma, \sigma^{\prime}}$. For $\mu \neq 0$, this new evolution operator is not any more norm conserving and the rate of growth of the generating function will be used to characterize the asymptotic work fluctuations. From now on, we assume that the perturbation $h=h(t)$, and hence also the matrix $\boldsymbol{L}^{(\mu)}$, is time-periodic. We can thus write the solution of Eq. (7) after $n$ periods as

$$
G_{\sigma, \mu}(n \tau)=\sum_{\sigma^{\prime}= \pm 1}\left(Q^{n}\right)_{\sigma, \sigma^{\prime}} p_{\sigma^{\prime}}(0) .
$$

The matrix $\boldsymbol{Q}$ is the single-period propagator

$$
\boldsymbol{Q}=\overrightarrow{\exp } \int_{0}^{\tau} \boldsymbol{L}^{(\mu)}(t) d t
$$

where $\overrightarrow{\exp }$ stands for the time-ordered exponential.

Let $\lambda$ and $\lambda^{\prime}$, where $\lambda \geqslant \lambda^{\prime}$, denote the eigenvalues of $\boldsymbol{Q}$ with corresponding left and right eigenvectors $\langle\lambda|,\left\langle\lambda^{\prime}\right|$ and $|\lambda\rangle,\left|\lambda^{\prime}\right\rangle$, respectively. Since $Q^{n}=\lambda^{n}|\lambda\rangle\left|\left\langle\lambda\left|+\lambda^{\prime n}\right| \lambda^{\prime}\right\rangle\right|\left\langle\lambda^{\prime}\right|$, the asymptotic behavior of $G_{\mu}(n \tau)$ for large times or large $n$ is determined by the largest eigenvalue $\lambda$ of $\boldsymbol{Q}$ :

$$
\lim _{n \rightarrow \infty} \frac{1}{n} \ln G_{\mu}(n \tau)=\ln \lambda=\phi_{\mu},
$$

where

$$
\lambda=\frac{\operatorname{tr} \boldsymbol{Q}+\sqrt{[\operatorname{tr} \boldsymbol{Q}]^{2}-4 \operatorname{det} \boldsymbol{Q}}}{2} .
$$

We now consider the work per period $w=W / n$ and focus on the large deviation function of $P_{W=n w}$ for $n \rightarrow \infty$ defined by

$$
I_{w}=-\lim _{n \rightarrow \infty} \frac{1}{n} \ln P_{n w} .
$$

This function quantifies the probability of exponentially rare deviations of $W$ from its average value $\langle W\rangle=n\langle w\rangle$,

$$
P_{W=n w} \asymp e^{-n I_{w}} .
$$

Note that $\langle w\rangle$ corresponds to the minimum of $I_{w}$ to ensure a long time convergence of the work $W$ toward $n w$ [45]. Inserting Eq. (12) into Eq. (6), one finds

$$
G_{\mu} \asymp \int_{-\infty}^{\infty} n d w \exp \left[-n\left(I_{w}-\mu w\right)\right] .
$$

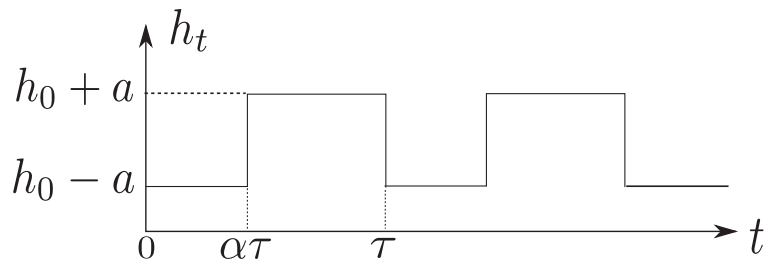

FIG. 1. External field following a piecewise constant protocol oscillating between the values $h_{0}-a$ to $h_{0}+a$ over a period of duration $\tau$.

The saddle point approximation with Eq. (10) leads to

$$
\phi_{\mu}=\max _{w}\left\{\mu w-I_{w}\right\} .
$$

In words, $\phi_{\mu}$ and $I_{w}$ are related one to each other by a Legendre transform. The evaluation of both the asymptotic generating function and the large deviation function are reduced to the calculation of the trace and determinant of the propagator $Q$, cf. (11).

\section{PIECEWISE CONSTANT DRIVING}

In order to evaluate analytically the propagator $Q$, we now consider the piecewise constant driving sketched on Fig. 1. This driving is characterized by four parameters: the intensity of the field $h_{0}$, the amplitude of the jumps $2 a$, the period of the driving $\tau$, and the cyclic ratio $\alpha$ (i.e., the fraction of time per period spent at the low value of the field).

Despite its apparent simplicity, the piecewise constant driving display discontinuities producing Dirac delta contributions in $\dot{h}$. We explain in the appendix how to deal with the discontinuities of the protocol to obtain $\boldsymbol{Q}$ and thus the work statistics. The final result reads:

$$
\begin{gathered}
\operatorname{tr} \boldsymbol{Q}=A \cosh \{2 a(2 \mu+1)\}+B, \\
\operatorname{det} \boldsymbol{Q}=C,
\end{gathered}
$$

where $A, B$, and $C$ are constants independent of $\mu$

$$
\begin{aligned}
& A=\frac{\left(1-z^{+}\right)\left(1-z^{-}\right)}{\cosh 2 a+\cosh 2 h_{0}}, \\
& B=\frac{\left(1+z^{+} z^{-}\right) \cosh 2 h_{0}+\left(z^{+}+z^{-}\right) \cosh 2 a}{\cosh 2 a+\cosh 2 h_{0}}, \\
& C=z^{+} z^{-},
\end{aligned}
$$

$$
\begin{aligned}
& \text { with } \\
& \qquad \begin{aligned}
z^{-} & =\exp \left(-\alpha \tau \omega^{-}\right), \\
z^{+} & =\exp \left[-(1-\alpha) \tau \omega^{+}\right], \\
\omega^{\epsilon} & =2 \omega\left(h_{0}+\epsilon a\right) \cosh \left(h_{0}+\epsilon a\right) \text { with } \epsilon=0, \pm .
\end{aligned}
\end{aligned}
$$

The crucial point to note is that the dependence on $\mu$ via the expression $\cosh \{2 a(2 \mu+1)\}$ is relatively simple. This feature can be exploited when performing the Legendre transform $I_{w}=\max _{\mu}\left\{\mu w-\phi_{\mu}\right\}$ and leads to (see Appendix for details)

$$
I_{w}=-\frac{w}{2}+\ln \left[\frac{2\left(x_{w}+\sqrt{\left(x_{w}\right)^{2}-1}\right)^{|w| / 4 a}}{A x_{w}+B+\sqrt{\left(A x_{w}+B\right)^{2}-4 C}}\right],
$$




$$
\begin{aligned}
& \text { with } \\
& \qquad \begin{aligned}
x_{w}= & -\frac{B w^{2}}{A\left(w^{2}-16 a^{2}\right)} \\
& -\frac{\sqrt{B^{2} w^{4}-\left(w^{2}-16 a^{2}\right)\left[w^{2}\left(B^{2}-4 C\right)+16 A^{2} a^{2}\right]}}{A\left(w^{2}-16 a^{2}\right)} .
\end{aligned}
\end{aligned}
$$

The explicit expressions for the asymptotic work generating function, Eq. (11) with Eqs. (16) and (17), and for the large deviation function, Eq. (20), are the main results of this paper.

\section{DISCUSSION}

In its traditional formulation, the Crooks fluctuation theorem applies to systems initially at equilibrium and is valid for any time. It connects the work fluctuations arising when applying an arbitrary protocol to those of a different experiment where the time-reversed protocol is considered. This result is a special case of the universal detailed fluctuation theorem for entropy production [46-51]. Indeed, when a system is in contact with a single reservoir, entropy production is given by the work minus the change in nonequilibrium free energy of the system. This latter reduces to the difference of equilibrium free energy in the traditional Crooks formulation. For a periodic driving, the change in nonequilibrium free energy over a period becomes zero when initial transients are gone and thus plays no role in the long time limit. Furthermore, for our piecewise constant modulation, the time-reversed driving is identical to the forward driving up to a time-shift, which again plays no role in the long time limit. As a result, the detailed fluctuation theorem for entropy production becomes a Crooks-like work fluctuation theorem for long times of the form $P_{W} / P_{-W}=$ $\exp \{W\}$. More precisely, the large deviation function and the work generating function satisfy the fluctuation theorem symmetry

$$
\begin{gathered}
I_{w}-I_{-w}=-w, \\
\phi_{\mu}=\phi_{-1-\mu} .
\end{gathered}
$$

These relations are easily verified. The second term in the large deviation function of Eq. (20) is even in $w$, and the first term immediately reproduces Eq. (22). For the work generating function, the $\mu$ dependency of $\phi$ appears only through the function $\cosh [2 a(1+2 \mu)]$ in Eq. (16), which is indeed invariant under the exchange of $\mu$ with $-1-\mu$. The detailed fluctuation theorem implies a Jarzynski-like integral fluctuation theorem, which for the generating function reads $\phi_{-1}=0$. Both the detailed and the integral fluctuation theorem are satisfied on Figs. 2 and 3, where $\phi_{\mu}$ and $I_{w}$ are plotted for various values of the protocol parameters and for Arrhenius, Fermi, and Bose rates.

These plots reveal a number of other features, which can be verified via analytical calculations (see Table I). First, due to the finite support of the large deviation function, the generating function displays a linear asymptotic behavior for $\mu \rightarrow \pm \infty$. The physical origin of this finite support is the existence of an upper and lower bound for the work per period, namely $\pm 4 a$ ( $\pm 2 a$ for every jump in the field). Second, the work variance (i.e., the width of the large deviation function) typically increases as the average number of jumps per period increases; see Fig. 4, where we observe, as the average number
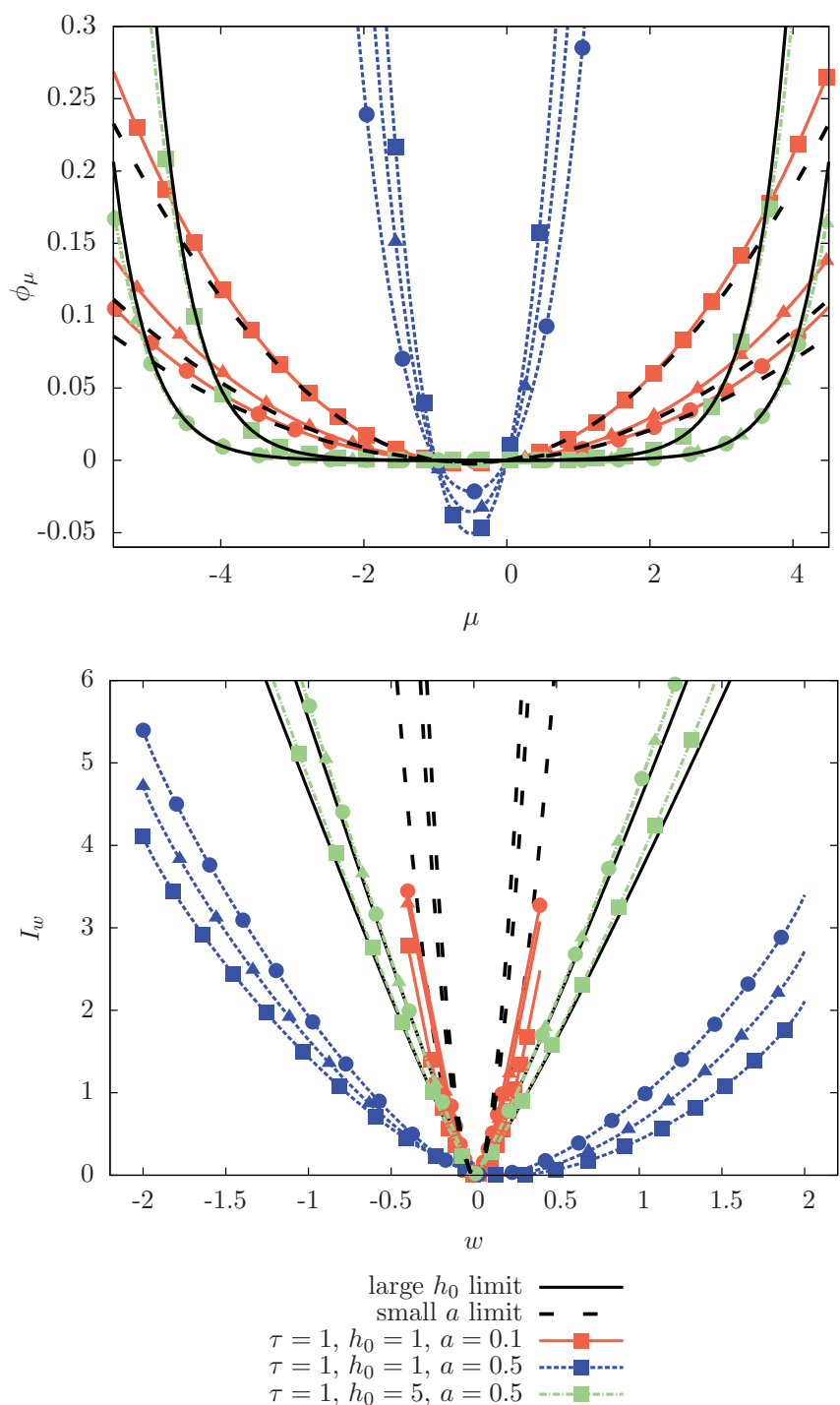

FIG. 2. (Color online) (Top) Cumulant generating function $\phi_{\mu}$ of work per period as a function of the Laplace parameter $\mu$. (Bottom) Large deviation function $I_{w}$ versus work per period. Various values of the field are plotted: high field, $h_{0}=5$ and $a=0.5$ (light green dotted dashed line); intermediate field, $h_{0}=1$ and $a=0.5$ (blue dashed line); and low amplitude of the driving, $h_{0}=1$ and $a=0.1$ (orange solid line). Symbols encode the types of rates: Arrhenius (squares), Bose (triangles), and Fermi (circle). The other parameters are $\tau=1$ and $\alpha=0.3$.

of jumps decreases from Arrhenius over Bose to Fermi rates, a corresponding decrease in the variance. Third, in the limit of infinite period, $\tau \rightarrow \infty$, the system has time to relax to the prevailing equilibrium distribution after each jump in the field. In this case, the work distribution becomes independent of the types of rates $\omega(h)$ since they all lead to the same equilibrium distribution, cf. (3). Since the field undergoes jumps, we are, however, not in a close-to-equilibrium regime. Fourth, the latter regime is reached in the limit of small jumps $a \rightarrow 0$, where the work distribution becomes Gaussian

$$
I_{w}=\frac{(w-\langle w\rangle)^{2}}{4\langle w\rangle} .
$$



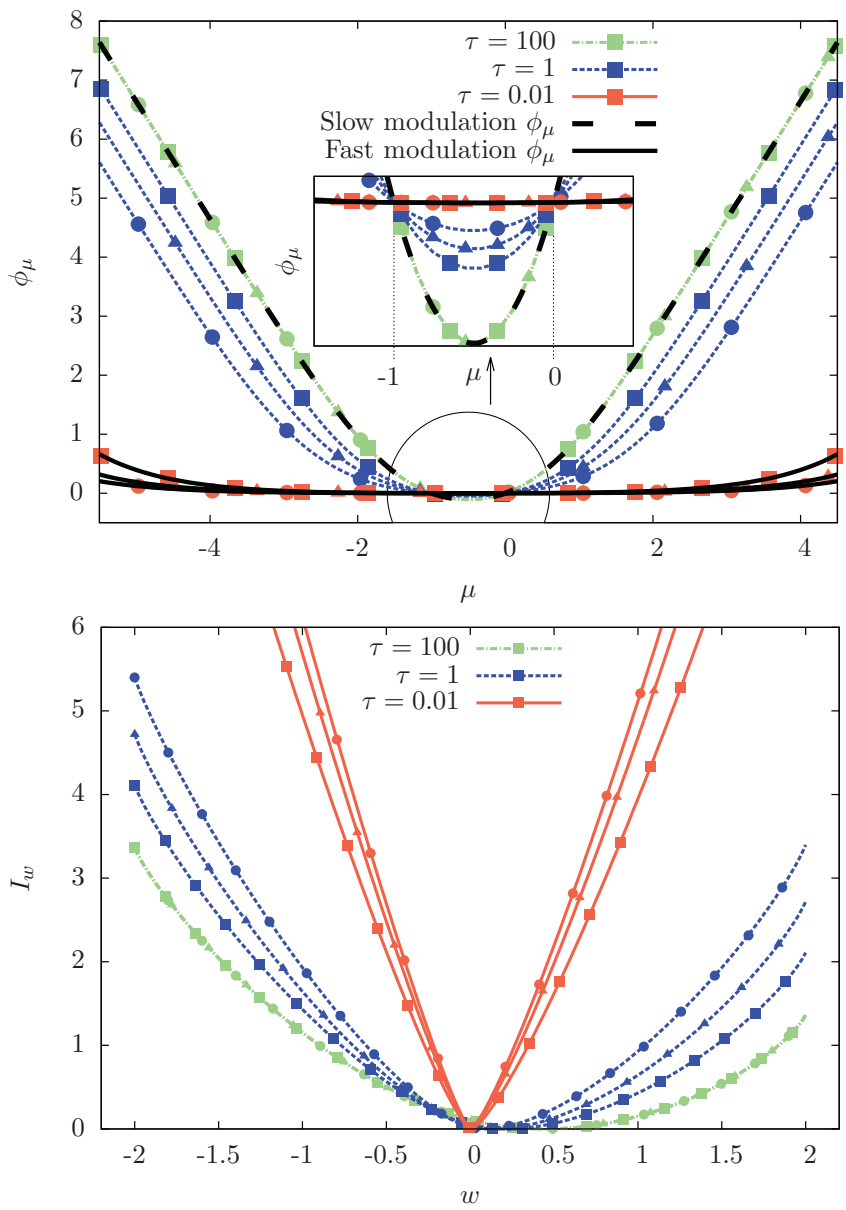

FIG. 3. (Color online) (Top) Cumulant generating function $\phi_{\mu}$ for the work per period versus the Laplace parameter $\mu$. (Bottom) Large deviation function $I_{w}$ versus work per period for various characteristic time scales of the driving: $\tau=100$ (light green dotted dashed line), $\tau=1$ (blue dashed line), and $\tau=0.01$ (orange solid line). Same symbols code as described in the legend of Fig. 2. Here, $a=0.5$, $h_{0}=1$, and $\alpha=0.3$.

The fact that the variance equals twice the average work is the signature of the fluctuation theorem for Gaussian processes. The general form of the average work $\langle w\rangle=\left.\partial_{\mu} \phi_{\mu}\right|_{\mu=0}$ is
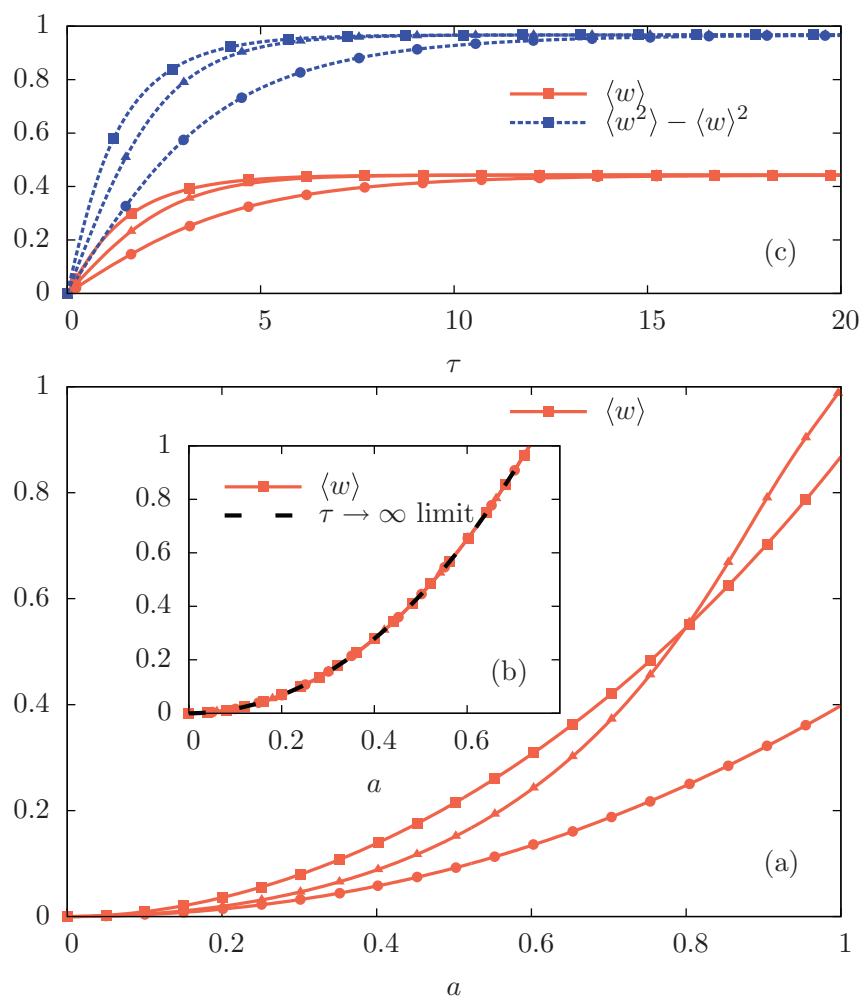

FIG. 4. (Color online) Average and variance of work per period as a function of the modulation amplitude $a$ for (a) and (b) or as a function of the period $\tau$ for (c). Parameters are $\alpha=0.3, h_{0}=1$, with $\tau=1$ for (a), $\tau=100$ for inset (b), and $a=0.5$ for (c). The symbol code is the same as described in the legend of Fig. 2.

given by

$\langle w\rangle=\frac{4 a \sinh (2 a)\left(1-e^{-(1-\alpha) \tau \omega^{+}}\right)\left(1-e^{-\alpha \tau \omega^{-}}\right)}{\left(\cosh \left(2 h_{0}\right)+\cosh (2 a)\right)\left(1-e^{-\alpha \tau \omega^{-}-(1-\alpha) \tau \omega^{+}}\right)}$,

and is plotted on Fig. 4. In the close-to-equilibrium regime, it becomes a quadratic function in the perturbation amplitude $a$. Fifth, a far-from-equilibrium regime is reached in the limit of fast modulation $\tau \rightarrow 0$ or large field $h_{0} \rightarrow \infty$. In both cases, the time scale of spin flips is much longer than a period. Consequently, the number of spin flips per period becomes

TABLE I. Cumulant generating function of work per period $\phi_{\mu}$ in various limits: Fast and slow modulation of the driving field $(\Gamma \tau \ll 1$ and $\Gamma \tau \gg 1)$, small amplitude of change in the field $(a \ll 1)$, and large values of the field $\left(h_{0} \gg 1\right)$. Most of these expansions are valid when the Laplace parameter $\mu$ remains inside a given interval depending on the expansion parameter.

\begin{tabular}{lc}
\hline \hline$\tau \ll 1$ & $\frac{\tau}{2}\left[-\alpha \omega^{-}-(1-\alpha) \omega^{+}+\sqrt{\left.\left(\alpha \omega^{-}+(1-\alpha) \omega^{+}\right)^{2}+4 \alpha(1-\alpha) \omega^{+} \omega^{-} \frac{\cosh 2 a(2 \mu+1)-\cosh 2 a}{\left[\cosh 2 a+\cosh 2 h_{0}\right]}\right]}\right.$ \\
$\Gamma \tau \gg 1$ & $\ln \frac{\cosh 2 h_{0}+\cosh 2 a(2 \mu+1)}{\cosh 2 a+\cosh 2 h_{0}}+\left(e^{-(1-\alpha) \tau \omega^{+}}+e^{-(1-\alpha) \tau \omega^{+}}\right) \frac{\cosh 2 a-\cosh 2 a(2 \mu+1)}{\cosh 2 h_{0}+\cosh 2 a(2 \mu+1)}$ \\
$a \ll 1$ & $\mu(1+\mu)\langle w\rangle=8 a^{2} \mu(1+\mu) \frac{\left(1-e^{-(1-\alpha) \tau \omega^{0}}\right)\left(1-e^{-\alpha \tau \omega^{0}}\right)}{\left(1-e^{-\tau \omega^{0}}\right)\left(1+\cosh 2 h_{0}\right)}$ \\
$h_{0} \gg a$ & Fermi and Bose rates \\
\hline
\end{tabular}


small. The work distribution converges to a Dirac delta distribution corresponding to a vanishing work per period.

\section{CONCLUSION}

The two-level system has played a crucial role in statistical physics to reveal the properties of both equilibrium and nonequilibrium systems. In the present paper, we derived an exact analytic expression for the (asymptotic) work distribution and work generating function of a two-level system in contact with a single thermal heat bath and subjected to a periodic piecewise constant field. We also showed that the universal fluctuation theorem for entropy production reduces to a corresponding Crooks-like fluctuation theorem for work. Our study could be easily extended to more complicated situations. The case of several heat baths is of obvious relevance since it would allow us to discuss the way in which the field modifies the energy transfers between the various baths. One could also increase the number of field states in the piecewise driving to break the asymptotic time-reversal symmetry $P=\bar{P}$ of the present study.

\section{ACKNOWLEDGMENT}

G.V. and M.E. are supported by the National Research Fund, Luxembourg under Project No. FNR/A11/02.

\section{APPENDIX} by

We denote the propagator for the system probabilities $p_{\sigma}(t)$

$$
\boldsymbol{U}\left(t, t_{0}\right)=\overrightarrow{\exp } \int_{t_{0}}^{t} \boldsymbol{L}\left(t^{\prime}\right) d t^{\prime} .
$$

Then, the generating function over a period reads

$$
\boldsymbol{Q}=\boldsymbol{K}^{(-2 a)} \boldsymbol{U}(\tau, \alpha \tau) \boldsymbol{K}^{(2 a)} \boldsymbol{U}(\alpha \tau, 0),
$$

where $\boldsymbol{K}^{( \pm 2 a)}$ is the operator cumulating the work over the jumps of amplitude $\pm 2 a$ in the protocol. $\boldsymbol{K}^{( \pm 2 a)}$ is obtained by taking the time-ordered exponential of the generator $\boldsymbol{L}^{(\mu)}$ between time $t^{-}$and $t^{+}$just before and after a jump at time $t$,

$$
\boldsymbol{K}^{( \pm 2 a)}=\overrightarrow{\exp } \int_{t^{-}}^{t^{+}}\left[\boldsymbol{L}\left(t^{\prime}\right)-\mu \boldsymbol{\sigma}_{z} \dot{h}\left(t^{\prime}\right)\right] d t^{\prime},
$$

with $\left(\sigma_{z}\right)_{\sigma, \sigma^{\prime}}=\sigma \delta_{\sigma, \sigma^{\prime}}$ and $\dot{h}\left(t^{\prime}\right)= \pm 2 a \delta\left(t-t^{\prime}\right)$. For $t^{-}$infinitely close to $t^{+}$, the propagator over a jump of amplitude $h$ is

$$
K_{\sigma, \sigma^{\prime}}^{(h)}=e^{-\sigma \mu h} \delta_{\sigma, \sigma^{\prime}} .
$$

At a constant value of the field $h=h_{0}+\epsilon a$ with $\epsilon= \pm 1$, the propagator in Eq. (A2) simplifies to

$$
\begin{aligned}
U_{\sigma, \sigma^{\prime}}\left(t, t_{0}\right)= & \frac{e^{\sigma\left(h_{0}+\epsilon a\right)}}{2 \cosh \left(h_{0}+\epsilon a\right)}\left(1-e^{-\omega^{\epsilon}\left(t-t_{0}\right)}\right) \\
& +\delta_{\sigma, \sigma^{\prime}} e^{-\omega^{\epsilon}\left(t-t_{0}\right)} .
\end{aligned}
$$

Using Eqs. (A2), (A4), and (A5), a lengthy calculation leads to $Q$ and to the final explicit expression of the trace given in Eq. (16).

The determinant of the propagator over a period is much easier to obtain [52]. If we define

$$
\boldsymbol{Q}(t)=\overrightarrow{\exp } \int_{0}^{t} \boldsymbol{L}^{(\mu)}\left(t^{\prime}\right) d t^{\prime},
$$

we see that det $Q(t)$ obeys a closed evolution equation

$$
\partial_{t} \operatorname{det} \boldsymbol{Q}(t)=\operatorname{tr}\left(\boldsymbol{L}^{(\mu)}(t)\right) \operatorname{det} \boldsymbol{Q}(t),
$$

which leads to

$$
\operatorname{det} \boldsymbol{Q}=\exp \left(\int_{0}^{\tau} \operatorname{tr} \boldsymbol{L}^{(\mu)}(t) d t\right)=z^{-} z^{+} .
$$

We now turn to the large deviation function, i.e., to the Legendre transform of the cumulant generating function

$$
\phi_{x}=\ln \left[A x+B+\sqrt{(A x+B)^{2}-4 C}\right]-\ln 2,
$$

where the $\mu$ dependence is hidden in the variable $x=$ $\cosh 2 a(2 \mu+1)$. Hence, the Legendre transform $I_{w}=$ $\max _{\mu}\left\{\mu w-\phi_{\mu}\right\}$ can be replaced by the extremum calculation in terms of $x, I_{w}=\max _{x}\left\{\mu_{x} w-\phi_{x}\right\}$, where $\mu_{x}=$ $\pm \operatorname{argcosh} x / 4 a-1 / 2$. The resulting equation for $x$ as a function of $w$ turns out to be quadratic, with the proper solution for $x$ given in Eq. (21). The large deviation function of Eq. (20) is found by evaluating $\mu_{x} w-\phi_{x}$ in $x_{w}$ using the logarithmic representation of the hyperbolic cosine function.
[1] C. Jarzynski, Phys. Rev. Lett. 78, 2690 (1997).

[2] C. Jarzynski, Phys. Rev. E 73, 046105 (2006).

[3] G. E. Crooks, Phys. Rev. E 61, 2361 (2000).

[4] R. Chelli, J. Chem. Phys. 130, 054102 (2009).

[5] R. J. Harris and G. M. Schütz, J. Stat. Mech. (2007) P07020.

[6] L. Y. Chen, J. Chem. Phys. 129, 091101 (2008).

[7] R. Lua and A. Grosberg, J. Phys. Chem. B 109, 6805 (2005).

[8] T. Speck and U. Seifert, Phys. Rev. E 70, 066112 (2004).

[9] H. C. Fogedby and A. Imparato, J. Stat. Mech. (2012) P04005.

[10] J. Farago, J. Stat. Phys. 107, 781 (2002).

[11] R. Chakrabarti, Pramana 72, 665 (2009).

[12] J. Hoppenau and A. Engel, J. Stat. Mech. (2013) P06004.
[13] A. Pal and S. Sabhapandit, Phys. Rev. E 87, 022138 (2013).

[14] R. van Zon and E. G. D. Cohen, Phys. Rev. E 67, 046102 (2003).

[15] A. Imparato and L. Peliti, Phys. Biol. 6, 025011 (2009).

[16] T. Mai and A. Dhar, Phys. Rev. E 75, 061101 (2007).

[17] S. Sabhapandit, Europhys. Lett. 96, 20005 (2011).

[18] P. Visco, A. Puglisi, A. Barrat, E. Trizac, and F. Wijland, J. Stat. Phys. 125, 533 (2006).

[19] I. Bena, C. V. den Broeck, and R. Kawai, Europhys. Lett. 71, 879 (2005).

[20] B. Cleuren, C. Van den Broeck, and R. Kawai, Phys. Rev. Lett. 96, 050601 (2006).

[21] A. Imparato and L. Peliti, Europhys. Lett. 70, 740 (2005). 
[22] A. Imparato and L. Peliti, Phys. Rev. E 72, 046114 (2005).

[23] N. Kumar, C. Van den Broeck, M. Esposito, and K. Lindenberg, Phys. Rev. E 84, 051134 (2011).

[24] S. Schuler, T. Speck, C. Tietz, J. Wrachtrup, and U. Seifert, Phys. Rev. Lett. 94, 180602 (2005).

[25] D. Collin, F. Ritort, C. Jarzynski, S. B. Smith, I. J. Tinoco, and C. Bustamante, Nature (London) 437, 231 (2005).

[26] I. Junier, A. Mossa, M. Manosas, and F. Ritort, Phys. Rev. Lett. 102, 070602 (2009).

[27] F. Douarche, S. Ciliberto, and A. Petrosyan, J. Stat. Mech. (2005) P09011.

[28] S. Joubaud, G. Huillard, A. Petrosyan, and S. Ciliberto, J. Stat. Mech. (2009) P01033.

[29] D. Andrieux, P. Gaspard, S. Ciliberto, N. Garnier, S. Joubaud, and A. Petrosyan, J. Stat. Mech. (2008) P01002.

[30] J. R. Gomez-Solano, L. Bellon, A. Petrosyan, and S. Ciliberto, Europhys. Lett. 89, 60003 (2010).

[31] L. Granger, M. Niemann, and H. Kantz, J. Stat. Mech. (2010) P06029.

[32] M. Einax and P. Maass, Phys. Rev. E 80, 020101 (2009).

[33] A. Saha and A. M. Jayannavar, Phys. Rev. E 77, 022105 (2008).

[34] R. Marathe and A. Dhar, Phys. Rev. E 72, 066112 (2005).

[35] F. Ritort, J. Stat. Mech. (2004) P10016.

[36] E. Subrt and P. Chvosta, J. Stat. Mech. (2007) P09019.

[37] M. Esposito, U. Harbola, and S. Mukamel, Phys. Rev. E 76, 031132 (2007).
[38] A. Bérut, A. Arakelyan, A. Petrosyan, S. Ciliberto, R. Dillenschneider, and E. Lutz, Nature (London) 483, 187 (2012).

[39] Y. Jun and J. Bechhoefer, Phys. Rev. E 86, 061106 (2012).

[40] D. Wu, K. Ghosh, M. Inamdar, H. J. Lee, S. Fraser, K. Dill, and R. Phillips, Phys. Rev. Lett. 103, 050603 (2009).

[41] C. Tietz, S. Schuler, T. Speck, U. Seifert, and J. Wrachtrup, Phys. Rev. Lett. 97, 050602 (2006).

[42] U. Seifert, Rep. Prog. Phys. 75, 126001 (2012).

[43] M. Esposito, Phys. Rev. E 85, 041125 (2012).

[44] P. Talkner, New J. Phys. 1, 4 (1999).

[45] H. Touchette, Phys. Rep. 478, 1 (2009).

[46] B. Shargel and T. Chou, J. Stat. Phys. 137, 165 (2009).

[47] U. Seifert, Phys. Rev. Lett. 95, 040602 (2005).

[48] M. Esposito and C. Van den Broeck, Phys. Rev. Lett. 104, 090601 (2010).

[49] G. Verley, R. Chétrite, and D. Lacoste, Phys. Rev. Lett. 108, 120601 (2012).

[50] G. Verley and D. Lacoste, Phys. Rev. E 86, 051127 (2012).

[51] C. Van den Broeck, in Proceedings of the International School of Physics “Enrico Fermi," Physics of Complex Colloids Vol. 184, edited by C. Bechinger, F. Sciortino, and P. Ziherl (IOS Press, Amsterdam, 2013).

[52] L. Y. Adrianova, Introduction to Linear systems of Differential Equations (American Mathematical Society, Providence, Rhode Island, 1995). 